\title{
BMJ Open Re-evaluation of potential predictors of calretinin and mesothelin in a population-based cohort study using assays for the routine application in clinical medicine
}

Swaantje Casjens (D , , ${ }^{1}$ Georg Johnen, ${ }^{1}$ Irina Raiko, ${ }^{1}$ Beate Pesch, ${ }^{1}$ Dirk Taeger, ${ }^{1}$ Carmen Töpfer, ${ }^{1}$ Sandra Schonefeld, ${ }^{1}$ Susanne Moebus, ${ }^{2}$ Karl-Heinz Jöckel, ${ }^{2}$ Thomas Brüning, ${ }^{1}$ Daniel Weber ${ }^{1}$

To cite: Casjens S, Johnen G, Raiko I, et al. Re-evaluation of potential predictors of calretinin and mesothelin in a populationbased cohort study using assays for the routine application in clinical medicine. BMJ Open 2021;11:e039079. doi:10.1136/ bmjopen-2020-039079

- Prepublication history and supplemental material for this paper is available online. To view these files, please visit the journal online (http://dx.doi. org/10.1136/bmjopen-2020039079).

Received 03 April 2020 Revised 06 October 2020 Accepted 15 October 2020

Check for updates

(C) Author(s) (or their employer(s)) 2021. Re-use permitted under CC BY-NC. No commercial re-use. See rights and permissions. Published by BMJ.

${ }^{1}$ Institute for Prevention and Occupational Medicine of the German Social Accident Insurance, Institute of the Ruhr University Bochum (IPA), Bochum, Germany

${ }^{2}$ Institute of Medical Informatics, Biometry and Epidemiology, University Duisburg-Essen, Essen, Germany

Correspondence to Dr Swaantje Casjens; casjens@ipa-dguv.de

\section{ABSTRACT}

Objectives Calretinin and mesothelin are molecular markers for the detection of malignant mesothelioma at early stages. Our objective was the re-evaluation of factors influencing calretinin and mesothelin concentrations in plasma of cancer-free men in order to minimise falsepositive tests when using commercial assays approved for clinical diagnostics.

Setting This re-evaluation used data and archived blood samples of the population-based Heinz Nixdorf Recall Study (HNRS) collected from 2011 to 2014.

Participants The present analysis comprised of 569 cancer-free men at the time of blood sampling (median age 70 years) from HNRS.

Primary and secondary outcomes Mesothelin plasma concentration was determined using ELISA and CLEIA (chemiluminescent enzyme immunoassay). Calretinin plasma concentration was assessed using ELISA. Results Compared with the previous determination of concentrations, we detected less false-positive tests using the commercial assays. In this analysis, we found nine false-positive calretinin tests using the ELISA (specificity $98.4 \%, 95 \% \mathrm{Cl} 97.0 \%$ to $99.2 \%$ ) and 24 false-positive mesothelin tests using both ELISA and CLEIA (specificity $95.8 \%, 95 \% \mathrm{Cl} 93.8 \%$ to $97.2 \%$ ). We confirmed renal dysfunction as major predictor of elevated marker concentrations. Mesothelin was additionally affected by bronchitis. Furthermore, elevated inflammation values and hypertension only affected the mesothelin concentration determined by ELISA.

Conclusions The newly available assays of calretinin and mesothelin approved for clinical diagnostics showed high specificities in the population-based cohort of elderly men without a malignant disease. The current evaluation provides a basis to consider influencing factors in order to further improve the diagnostic procedure.

\section{INTRODUCTION}

Malignant mesothelioma is an aggressive cancer of the serous membranes mainly caused by a former exposure to asbestos.
Strengths and limitations of this study

- This is the first study exploring potential predictors associated with false-positive tests of blood-based markers for malignant mesothelioma in plasma using assays approved for clinical diagnostics.

- The study population was enrolled from the general population and not from a cohort of asbestos workers.

- Determinants influencing marker concentrations were identified in order to minimise false-positive tests of calretinin and mesothelin for the use of these markers in clinical practice to detect malignant mesothelioma at early stages.

- Due to the higher incidence of mesothelioma in men compared with women, only men were included in this study, so possible gender differences could not be investigated.

- High specificities of mesothelin and calretinin were based on small numbers of false-positive tests limiting the power of detecting predictors.

Worldwide its incidence is increasing and with 38000 annual deaths mesothelioma presents a global health issue. ${ }^{1}$ Mesothelioma is characterised by a very long latency period between exposure to asbestos and diagnosis and a very poor prognosis with a median survival being less than 12 months because the majority of patients are diagnosed at advanced stages. ${ }^{23}$ It has been shown that patients diagnosed earlier and undergoing multimodal therapy have longer median survival rates in the range of 30-51 months. ${ }^{4}$ Thus, the detection of mesothelioma at early stages seems to be a promising opportunity to improve therapy options and survival.

Currently, calretinin and mesothelin are the most prominent blood-based biomarkers 
for mesothelioma. ${ }^{56}$ Notably, imaging is less sensitive in detecting this malignant disease. ${ }^{7}$ For the detection of mesothelioma prior to a clinical diagnosis, only the combination of calretinin and mesothelin has been validated in a prospective study so far, showing a sensitivity of $46 \%$ at a predefined specificity of $98 \%$ using plasma samples from prediagnostic mesothelioma cases. ${ }^{8}$ This high specificity is necessary in screening with tumour markers to reduce false-positive tests in cancer-free subjects to an acceptable minimum, ${ }^{9}$ because false-positive tests might result in psychological stress and needless invasive diagnostic work-up procedures for the individuals concerned. Thus, there is an urgent need to identify parameters influencing the marker concentrations in subjects without the malignant disease to improve the performance of the markers for the routine application in clinical practice. It is known that renal dysfunction ${ }^{10-13}$ and hypertension ${ }^{13}$ could lead to increased mesothelin concentrations. Also calretinin might be influenced by renal dysfunction. ${ }^{13}$

This study re-evaluates a previous analysis of calretinin and mesothelin in plasma samples from 569 cancer-free men to identify factors influencing their concentrations in order to minimise false-positive test results for the detection of malignant mesothelioma. ${ }^{13}$ In contrast to the previous analysis, here we only used commercially available assays, which are approved for clinical diagnostics, namely a calretinin ELISA, a mesothelin ELISA and a mesothelin CLEIA (chemiluminescent enzyme immunoassay), to examine predictors of positive tests in order to further improve the specificity of these markers.

\section{METHODS}

\section{Study population}

The study population comprised of 569 men from the second follow-up survey (2011-2014) of the Heinz Nixdorf Recall Study (HNRS) within the framework of 'Arbeitsmedizinische Forschung in epidemiologischen Kohortenstudien'-Occupational medical research in epidemiological cohort studies, which aimed at investigating occupational risk factors within HNRS. ${ }^{14}$ HNRS is a prospective population-based cohort study in the Ruhr area, an industrial centre in Germany. Its rationale, design and implementation have been described before. ${ }^{15}$ Notably, at the time of blood collection, all participants were without a malignant disease.

Information on sociodemographic characteristics, current and chronic diseases (hypertension, bronchial asthma, bronchitis, chronic obstructive pulmonary disease, pneumoconiosis, pneumonia, pulmonary emphysema, tuberculosis, arthritis, diabetes mellitus, hepatitis and cancer), and medications was derived from questionnaires. We further included follow-up information on the development of cancer and data on 36 blood parameters analysed in the central laboratory of the University Hospital Essen with standard methods. ${ }^{16}$ The distribution of blood parameters in the study population and standard values have already been published in our previous study (online supplemental table S1). ${ }^{13}$

\section{Determination of mesothelin and calretinin}

Peripheral blood was collected from each participant in $9.0 \mathrm{~mL}$ S-Monovette EDTA gel tubes (Sarstedt, Nümbrecht, Germany) and centrifuged at $2000 \times \mathrm{g}$ for 10 min. Plasma was separated from the cellular fraction and frozen immediately at $-80^{\circ} \mathrm{C}$ until analyses. Calretinin was measured between October 2018 and November 2018. Mesothelin was determined between September 2019 and October 2019 (CLEIA) and October 2019 and November 2019 (ELISA), respectively.

Plasma calretinin was determined using the Calretinin ELISA kit (DLD Diagnostika, Hamburg, Germany) according to the manufacturers' instructions. The Calretinin ELISA is based on polyclonal anticalretinin antibodies from immunised rabbits. ${ }^{17}$ The limit of detection (LoD) of the Calretinin ELISA is $0.05 \mathrm{ng} / \mathrm{mL}$ and the overall precision $\leq 10.4 \% \mathrm{CV}$. Marker results were marked positive if the calretinin concentrations were above the cut-off of $0.6 \mathrm{ng} / \mathrm{mL}^{8}{ }^{8}$ Plasma mesothelin was measured with the Lumipulse G Mesothelin assays (Fujirebio, Tokyo, Japan) using the CLEIA analyser Lumipulse G600 II (Fujirebio) and the Mesomark ELISA kit (Fujirebio Diagnostics, Malvern, Pennsylvania, USA), respectively, according to the manufacturers' instructions. The LoD of the mesothelin CLEIA is $0.03 \mathrm{nM}$ and the overall precision $\leq 10.0 \% \mathrm{CV}$. The LoD of the mesothelin ELISA is $0.3 \mathrm{nM}$ and the overall precision $\leq 15.0 \% \mathrm{CV}$. Marker results were marked as positive if mesothelin concentrations were $\geq 1.5 \mathrm{nM}$ (mesothelin CLEIA) and $\geq 2.0 \mathrm{nM}$ (mesothelin ELISA), respectively. The mesothelin assays are based on the monoclonal antibodies OV569 and $4 \mathrm{H} 3 .{ }^{18}$ All marker kits are CE marked and approved for in vitro diagnostics.

\section{Statistical analysis}

Box plots with median and IQR were used to depict the distribution of marker concentrations. Whiskers represent minimum and maximum. Marker concentrations were compared using the non-parametric Kruskal-Wallis test. Specificity of markers was calculated as amount of negative marker results divided by amount of total marker results and presented with 95\% modified Wald confidence limits. Spearman's correlation coefficients $\left(\mathrm{r}_{\mathrm{S}}\right)$ and $95 \%$ CIs were used to describe rank correlations between variables.

As effect estimate for marker concentrations being above their cut-offs, we calculated prevalence ORs (PORs) and 95\% CIs. Potential predictors were age, smoking status (never, former, current), self-assessed diseases (yes, no), development of cancer after blood sampling (yes, no), current medication intake (yes, no) and 36 blood parameters (within, below or above standard values). First, PORs were estimated with univariate logistic regression models to identify influencing variables for each marker separately. Next, we conducted multiple regression models based on our previous analyses and preliminary work. ${ }^{13}$ 
Statistical analyses were done using SAS, V.9.4 (SAS Institute). GraphPad Prism V.7.04 (GraphPad Software, La Jolla, California, USA) was used to prepare graphs.

\section{Patient and public involvement}

Patients and/or the public were not involved in the design, or conduct, or reporting, or dissemination plans of this research.

\section{RESULTS}

\section{Study population}

The study population has been characterised elsewhere. ${ }^{13}$ In brief, the median age of the 569 analysed men was 70 years (range 56-84 years). Most men have ever smoked (12\% current smokers, $56 \%$ former smokers), whereas 176 men were non-smokers $(31 \%)$. After blood collection cancer other than mesothelioma was diagnosed in 20 men, whereof 9 received a diagnosis within 1 year. The most frequently self-reported diseases were hypertension with 365 cases $(64.1 \%)$ and diabetes mellitus with 120 cases $(21.1 \%)$. Hence, antihypertensive drugs were commonly used within the last week before examination $(61.5 \%)$. Most participants with hypertension took antihypertensive drugs $(95.9 \%)$.

\section{Marker performance}

The median calretinin concentration was $0.17 \mathrm{ng} / \mathrm{mL}$ (IQR 0.12-0.24 ng/mL) and showed nine false-positive marker tests $(1.6 \%)$. Hence, the specificity for calretinin was $98.4 \%$ (95\% CI $97.0 \%$ to $99.2 \%$ ). Median mesothelin concentrations were $0.66 \mathrm{nM}$ (IQR $0.49-0.94 \mathrm{nM}$ ) and $0.84 \mathrm{nM}$ (IQR $0.57-1.21 \mathrm{nM}$ ) using the mesothelin CLEIA and mesothelin ELISA, respectively. In both mesothelin assays, positive results were observed for 24 subjects $(4.2 \%)$, resulting in specificities of $95.8 \%$ (95\% CI 93.8\% to $97.2 \%)$. Twenty-one men $(3.7 \%)$ were tested marker positive with both assays. The distributions of all marker concentrations in the study group (online supplemental figure 1) and the comparisons with those of previously used marker assays (online supplemental figure 2) are presented in the online supplemental file.

The Spearman correlations between markers, age and blood parameters determined previously are presented in table 1. Calretinin and mesothelin were weakly correlated whereby the correlation of the calretinin ELISA with the mesothelin CLEIA was slightly stronger $\left(\mathrm{r}_{\mathrm{s}}=0.25,95 \%\right.$ CI 0.18 to 0.33$)$. The concentrations of calretinin and mesothelin increased by age and by blood parameters reflecting renal function (cystatin $\mathrm{C}$ and creatinine).

\section{Potential predictors of positive test results}

Table 2 depicts the results of the univariate logistic regression models for predictors of marker concentration above the cut-offs. Calretinin and mesothelin were affected by renal dysfunction as indicated by cystatin $\mathrm{C}$ and creatinine, as well as brain natriuretic peptide (BNP), haemoglobin, red blood cells and haematocrit. Additionally, mesothelin, determined by CLEIA as well as ELISA, was influenced by age, $\mathrm{C}$ reactive protein (CRP), fibrinogen, current hypertension, current antihypertensive intake and current bronchitis as shown by significantly increased PORs. Mesothelin determined using the ELISA was additionally affected by increased triglycerides concentrations, a marker of fat metabolism. Only one participant with cancer past blood collection showed an increased mesothelin concentration determined by CLEIA and no

Table 1 Spearman correlations $\left(R_{S}\right)$ and $95 \% \mathrm{Cl}$ of calretinin and mesothelin assessed with assays approved for clinical diagnostics with age and blood parameters determined previously

\begin{tabular}{|c|c|c|c|}
\hline & Calretinin ELISA (ng/mL) & Mesothelin CLEIA (nM) & Mesothelin ELISA (nM) \\
\hline & $r_{s}(95 \% \mathrm{Cl})$ & $r_{s}(95 \% \mathrm{Cl})$ & $r_{s}(95 \% \mathrm{Cl})$ \\
\hline Mesothelin CLEIA (nM) & $0.25(0.18$ to 0.33$)$ & & \\
\hline Age (years) & $0.21(0.13$ to 0.29$)$ & $0.27(0.20$ to 0.35$)$ & $0.24(0.16$ to 0.32$)$ \\
\hline $\mathrm{C}$ reactive protein $(\mathrm{mg} / \mathrm{dL})$ & $0.21(0.13$ to 0.29$)$ & $0.10(0.01$ to 0.18$)$ & 0.08 (0.00 to 0.16$)$ \\
\hline Cystatin C (mg/L) & $0.53(0.46$ to 0.58$)$ & $0.33(0.25$ to 0.40$)$ & $0.27(0.20$ to 0.35$)$ \\
\hline Creatinine (mg/dL) & 0.41 (0.34 to 0.47$)$ & $0.28(0.20$ to 0.35$)$ & $0.24(0.17$ to 0.32$)$ \\
\hline Brain natriuretic peptide (pg/mL) & $0.14(0.06$ to 0.22$)$ & $0.06(-0.02$ to 0.14$)$ & $0.06(-0.03$ to 0.14$)$ \\
\hline Haemoglobin, (g/L) & $-1.3(-0.21$ to -0.05$)$ & $-0.80(-0.16$ to 0.00$)$ & $-0.70(-0.15$ to 0.01$)$ \\
\hline Red blood cells $\left(\times 10^{12} / \mathrm{L}\right)$ & $-0.10(-0.19$ to -0.02$)$ & $-0.11(-0.19$ to -0.02$)$ & $-0.10(-0.18$ to -0.02$)$ \\
\hline
\end{tabular}

Statistically significant results are marked in bold.

CLEIA, chemiluminescent enzyme immunoassay. 


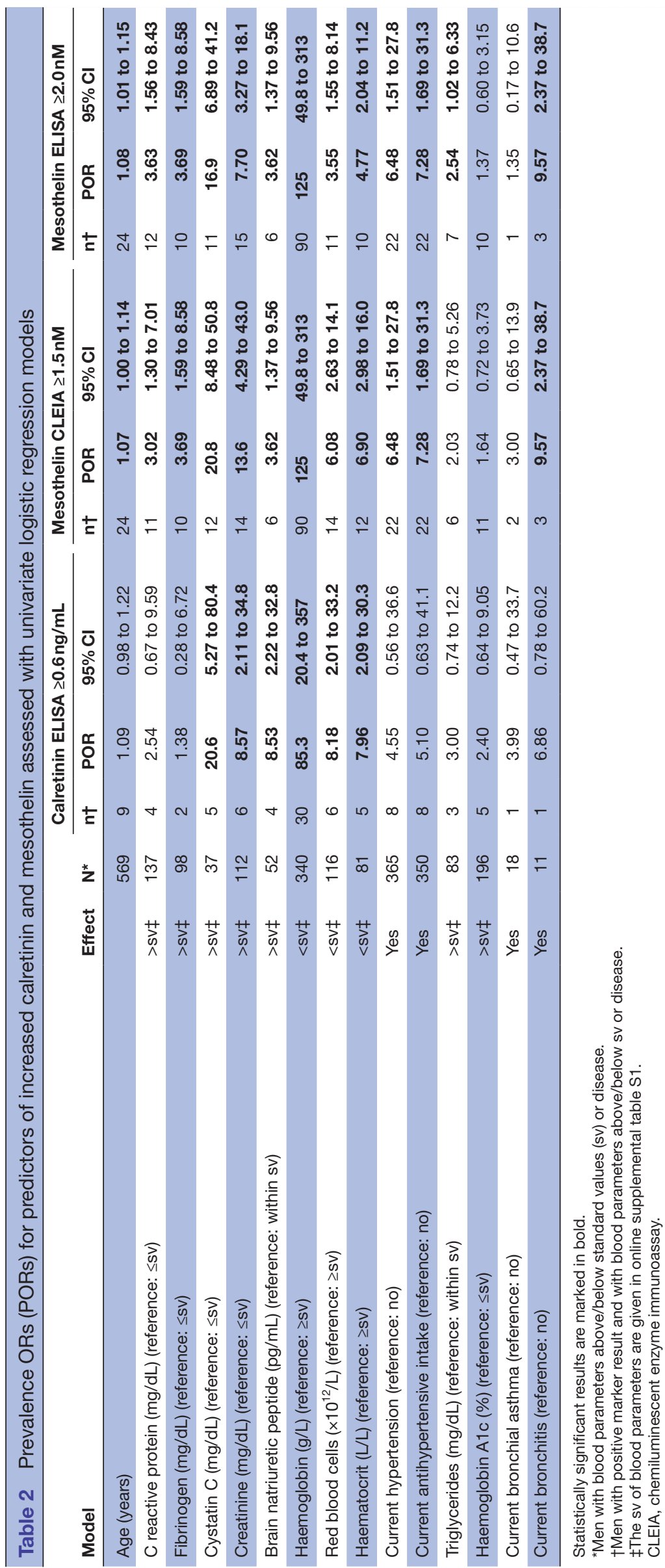


Table 3 Results of multiple logistic regression models for increased calretinin and mesothelin

\begin{tabular}{|c|c|c|c|c|c|c|c|c|c|c|}
\hline \multirow[b]{2}{*}{ Variable } & \multirow[b]{2}{*}{ Effect } & \multicolumn{3}{|c|}{ Calretinin ELISA $\geq 0.6 \mathrm{ng} / \mathrm{mL}$} & \multicolumn{3}{|c|}{ Mesothelin CLEIA $\geq 1.5 \mathrm{nM}$} & \multicolumn{3}{|c|}{ Mesothelin ELISA $\geq 2.0 \mathrm{nM}$} \\
\hline & & $\mathbf{n}^{*}$ & PORT & $95 \% \mathrm{Cl}$ & $\mathrm{n}^{*}$ & POR† & $95 \% \mathrm{Cl}$ & $\mathrm{n}^{*}$ & POR† & $95 \% \mathrm{Cl}$ \\
\hline Age (years) & & - & - & & 23 & 1.06 & 0.99 to 1.14 & 23 & 1.07 & 1.00 to 1.16 \\
\hline $\begin{array}{l}\text { C reactive protein (mg/dL) } \\
\text { (reference: } \leq \mathrm{sv} \ddagger \text { ) }\end{array}$ & $>s v \ddagger$ & - & - & & 11 & 2.25 & 0.87 to 5.84 & 12 & 3.00 & 1.17 to 7.67 \\
\hline $\begin{array}{l}\text { Current hypertension } \\
\text { (reference: no) }\end{array}$ & Yes & 8 & 3.00 & 0.36 to 25.3 & 21 & 4.40 & 0.95 to 20.4 & 21 & 4.63 & 1.01 to 21.2 \\
\hline $\begin{array}{l}\text { Current bronchitis } \\
\text { (reference: no) }\end{array}$ & Yes & - & - & & 3 & 6.13 & 1.10 to 34.1 & 3 & 7.38 & 1.35 to 40.3 \\
\hline
\end{tabular}

Statistically significant results are marked in bold.

*Men with positive marker result and with blood parameters above/below standard values (sv) or disease.

†Prevalence ORs (PORs) with 95\% Cl.

$\ddagger$ The sv of blood parameters are given in online supplemental table S1.

CLEIA, chemiluminescent enzyme immunoassay.

participant an increased calretinin concentration above the cut-off at the time of blood collection (data not shown).

The results of the multiple logistic regression models in the whole study population are presented in table 3. Calretinin concentration was only affected by cystatin $\mathrm{C}$ with a POR of 17.5 (95\% CI 4.40 to 69.7). Cystatin C (mesothelin CLEIA: POR=12.1, 95\% CI 4.46 to 33.0; mesothelin ELISA: POR $=9.58,95 \% \mathrm{CI} 3.46$ to 26.5 ) and current bronchitis (mesothelin CLEIA: POR $=6.13,95 \%$ CI 1.10 to 34.1 ; mesothelin ELISA: POR=7.38, 95\% CI 1.35 to 40.3) showed an impact on mesothelin. Whereas elevated inflammation values (CRP: $\mathrm{POR}=3.00,95 \% \mathrm{CI} 1.17$ to 7.67) and current hypertension ( $\mathrm{POR}=4.63,95 \%$ CI 1.01 to 21.2) only affected the mesothelin concentration determined by ELISA.

\section{DISCUSSION}

Cancer is frequently diagnosed at late stages of the disease when therapy options are limited. One major aim in cancer research is to detect cancer at early and hence potentially more curative stages. For malignant mesothelioma it is indicated that the determination of mesothelin and calretinin in plasma is appropriate for the detection of the tumour up to about a year before the clinical diagnosis. ${ }^{8}$ Importantly, in screening procedures, falsepositive tests in cancer-free individuals should be reduced to an acceptable minimum in order to avoid an invasive diagnostic work-up. ${ }^{9}{ }^{19}$ Knowledge regarding the impact of influencing factors, for example, benign diseases and specific blood parameters, leading to altered marker concentrations in subjects without malignant disease, is often limited. However, relevant predictors must be considered when subjects are examined with the goal to detect cancer early.

The move from bench to bedside in routine diagnostics might be hampered by a lack of reliable assays, because laboratory-developed assays are neither approved for clinical diagnostics nor commercially available and optimised to that end. This was particularly true for the laboratorydeveloped calretinin ELISA evaluated in our previous study. ${ }^{13}$ Additionally, the mesothelin ELISA evaluated in the previous study is for research use only and not approved for use in diagnostic procedures. ${ }^{13}$ In contrast, the calretinin ELISA, mesothelin ELISA and mesothelin CLEIA in this study fulfil both criteria. Furthermore, the mesothelin CLEIA is an automated assay, another important criterion for the application in routine diagnostics. Thus, it was necessary to re-evaluate the potential influencing factors on calretinin and mesothelin concentrations for clinical diagnostics to minimise false-positive tests.

Performing the measurements using the approved assays for calretinin (ELISA) and mesothelin (ELISA, CLEIA), we observed specificities of $98.4 \%$ and $95.8 \%$, respectively. Whereas the obtained specificity for mesothelin confirmed the results of the previous study, an increased specificity from $94.0 \%$ to $98.4 \%$ was observed for calretinin. ${ }^{13}$ Hence, the commercially available ELISA, which was based on the antibodies of the original laboratory-developed ELISA by Raiko $\mathrm{et} \mathrm{al},{ }^{17}$ is less susceptible to influencing factors and makes the marker more reliable for clinical diagnostics. Five men were presented with a positive calretinin ELISA and a positive mesothelin CLEIA test (99.1\% specificity) and only four men with a positive calretinin ELISA and a positive mesothelin ELISA test (99.3\% specificity), confirming the results of our previous study for the general population. ${ }^{13}$ However, the specificity in the target population, that is, subjects with a former exposure to asbestos and/or asbestos-related diseases, might be lower-as indicated by Cui et al. ${ }^{6}$ In our study, we initially chose to focus on men, because the risk to develop mesothelioma is higher in men than in women, due to a higher probability of an occupational 
exposure to asbestos. From the general population of Germany, a gender ratio of about 3.8 to 1 can be observed for the incidence of malignant mesothelioma. ${ }^{20}$ However, future studies including women are warranted.

Nevertheless, an advantage of the examined populationbased cohort is the comprehensive set of data on influencing factors in contrast to the more limited data recorded in clinical routine. ${ }^{9}$ This enables the identification of determinants with an impact on marker concentrations. It is well known that renal dysfunction affects the mesothelin concentration ${ }^{11-13}$ and this was confirmed in this study, as well as for calretinin. ${ }^{13}$ In more detail, we showed that indicators of renal dysfunction, namely cystatin $\mathrm{C}$ and creatinine, and BNP were increased whereas anaemia-related parameters (haemoglobin, red blood cells and haematocrit) were decreased in individuals with increased calretinin and mesothelin concentrations. Thus, to keep specificity in clinical diagnostics high, it might be meaningful to determine established markers of renal dysfunction, for example, cystatin $\mathrm{C}$ or creatinine in addition to the cancer markers. Notably, cystatin $\mathrm{C}$ might be superior to creatinine because it is not affected by age, gender and muscle mass. ${ }^{21}{ }^{22}$ In contrast, BNP, primarily a marker associated with heart failure, can also be increased by advanced renal dysfunction $^{23}$ and an increased concentration of BNP in pleural effusions has recently been associated with malignant mesothelioma. ${ }^{24}$ Hypertension is strongly associated with chronic kidney disease ${ }^{20}$ and was already indicated as an influencing factor for mesothelin. ${ }^{10}$ In this study, subjects with hypertension showed a fourfold higher chance of increased mesothelin, independently of the assay used, in comparison with non-hypertonic men when considering other factors as well. This effect is lower in comparison with the previous evaluation. ${ }^{13}$ Notably, using a higher cut-off of $2.9 \mathrm{nM}$ for the mesothelin ELISA, as suggested for the early diagnosis of mesothelioma in a high-risk cohort of asbestos workers, ${ }^{8}$ no major differences could be observed compared with the lower cut-off, only the effect of BNP did no longer exist (online supplemental table S2). However, hypertension could not be adequately analysed, because all participants with a positive marker result suffered from high blood pressure. Overall, hypertension is prevalent in elderly men.$^{25}$ However, calretinin was not affected by hypertension. This supports the determination of calretinin within the marker panel to detect mesothelioma at early stages.

No influence of age could be observed for calretinin, confirming previous results. ${ }^{13} 17{ }^{26}$ In contrast, a weak influence of age on mesothelin could be observed as indicated by a POR of 1.07 (95\% CI 1.00 to 1.14$)$ using the mesothelin ELISA and a POR of 1.08 (95\% CI 1.01 to 1.15) using the mesothelin CLEIA. However, published results regarding the association of age and mesothelin are still inconsistent. No differences of mesothelin concentrations regarding age could be observed by Pass et $a l,{ }^{27}$ whereas age was a statistical predictor of mesothelin in three recent studies. ${ }^{2628} 29$ Notably, using the higher cut-off as applied by Johnen $e t a l{ }^{8}$ the effect of age on mesothelin levels was no longer apparent, but the result was based on a small number of affected individuals. Nevertheless, it might be meaningful to consider age for the assessment of mesothelin results, for example, by using an age-depended cut-off.

In normal tissue, mesothelin is expressed at low levels and is not detectable. Usually, its release into serum results from malignant mesothelioma, but also by some other cancers (eg, pancreatic adenocarcinoma or ovarian cancers) but not from other inflammatory or pleural diseases. ${ }^{30-32}$ Here, bronchitis was marginally associated with increased mesothelin concentrations, confirming the results of our previous study. ${ }^{13}$ Thus, the current health status of the patients should be considered, when plasma samples are collected. In contrast to the previous study, ${ }^{13}$ bronchial asthma was not associated with increased calretinin or mesothelin concentrations.

Additionally, elevated concentrations of triglycerides in participants were marginally associated with increased mesothelin concentrations using the mesothelin ELISA. This is not necessarily in contrast to a previous study where triglycerides and other potentially interfering substances were added artificially to serum samples in order to determine possible disruptive effects on the performance of the assay. ${ }^{10}$ In our previous study and when using the mesothelin CLEIA, we did not observe an effect of triglycerides on mesothelin concentrations. Because these findings are based on small numbers of affected individuals and no other parameter of the fat metabolism (total high-density lipoprotein and lowdensity lipoprotein cholesterol) had an impact on elevated mesothelin concentrations (data not shown), this could also be a random finding.

In contrast to laboratory-developed assays, the use of commercially available ELISAs and CLEIAs enables a quality-assured measurement in the clinical routine of calretinin and mesothelin in at-risk groups in order to detect malignant mesothelioma at earlier stages. The results of this study additionally support the clinicians for a better assessment of the patients and obtained marker results in order to guide the subsequent proceedings. However, larger studies are needed to validate these results in order to potentially incorporate confounding marker values in a reliable formula to calculate the probability of a diagnosis of malignant mesothelioma.

Some limitations of our study are noteworthy. The high observed specificities of calretinin (98\%) and mesothelin $(95 \%)$ were based on small numbers of false positives which limit the power of detecting predictors. Only men were considered in this study, so possible gender differences could not be investigated. In addition, the study population was recruited from the general population and not from a cohort of asbestos workers with a higher prevalence of asbestosis. 


\section{CONCLUSIONS}

Both calretinin and mesothelin showed high specificities in this population-based cohort of cancer-free men. Whereas calretinin was only affected by renal dysfunction, mesothelin was affected by renal dysfunction as well as hypertension, age and current bronchitis. The application of biomarkers for early detection of mesothelioma requires reliable assays that have been approved for clinical diagnostics. The current evaluation of newly available assays for calretinin and mesothelin provides a basis to consider influencing factors in order to further improve the diagnostic procedure.

Acknowledgements We acknowledge the support of the DFG Open Access Publication Funds of the Ruhr University Bochum.

Contributors SC, GJ, BP, DT, SM, K-HJ, TB and DW conceived and designed the experiments and analyses. IR, CT and SS performed the experiments. SC analysed the data. SC and DW drafted the manuscript. All authors critically read, revised and approved the manuscript.

Funding The Institute for Medical Informatics, Biometry and Epidemiology was supported by the German Social Accident Insurance grant number FP 295.

Disclaimer The authors from the Institute for Prevention and Occupational Medicine of the German Social Accident Insurance-Institute of the Ruhr University Bochum (IPA) are independent from the German Social Accident Insurance in study design, access to the collected data, responsibility for data analysis and interpretation, and the right to publish. The views expressed in this paper are those of the authors and not necessarily those of the German Social Accident Insurance.

Competing interests The IPA has supplied DLD Diagnostika with the antibodies to produce the Calretinin ELISA kits. In turn, the IPA has received Calretinin ELISA kits at a reduced price and may benefit from future sales of the kits. The IPA has received Mesomark ELISA kits at a reduced price and free Lumipulse G Mesothelin assay reagents for the temporarily provided Lumipulse G600 II instrument from Fujirebio. Otherwise, the individual authors declare no competing interests.

\section{Patient consent for publication Not required}

Ethics approval Approval for the study was obtained from the ethical commission of the Medical Faculty of the University Duisburg-Essen (approval number 11-4678). All participants gave their written informed consent.

Provenance and peer review Not commissioned; externally peer reviewed. Data availability statement № additional data are available.

Supplemental material This content has been supplied by the author(s). It has not been vetted by BMJ Publishing Group Limited (BMJ) and may not have been peer-reviewed. Any opinions or recommendations discussed are solely those of the author(s) and are not endorsed by BMJ. BMJ disclaims all liability and responsibility arising from any reliance placed on the content. Where the content includes any translated material, BMJ does not warrant the accuracy and reliability of the translations (including but not limited to local regulations, clinical guidelines, terminology, drug names and drug dosages), and is not responsible for any error and/or omissions arising from translation and adaptation or otherwise.

Open access This is an open access article distributed in accordance with the Creative Commons Attribution Non Commercial (CC BY-NC 4.0) license, which permits others to distribute, remix, adapt, build upon this work non-commercially, and license their derivative works on different terms, provided the original work is properly cited, appropriate credit is given, any changes made indicated, and the use is non-commercial. See: http://creativecommons.org/licenses/by-nc/4.0/.

ORCID iD

Swaantje Casjens http://orcid.org/0000-0002-0148-8859

\section{REFERENCES}

1 Odgerel C-O, Takahashi K, Sorahan T, et al. Estimation of the global burden of mesothelioma deaths from incomplete national mortality data. Occup Environ Med 2017;74:851-8.
2 Hodgson JT, McElvenny DM, Darnton AJ, et al. The expected burden of mesothelioma mortality in Great Britain from 2002 to 2050. Br J Cancer 2005;92:587-93.

3 Mineo TC, Ambrogi V. Malignant pleural mesothelioma: factors influencing the prognosis. Oncology 2012;26:1164-75.

4 Wald O, Sugarbaker DJ. New concepts in the treatment of malignant pleural mesothelioma. Annu Rev Med 2018;69:365-77.

5 Johnen G, Gawrych K, Raiko I, et al. Calretinin as a blood-based biomarker for mesothelioma. BMC Cancer 2017;17:386.

6 Cui A, Jin X-G, Zhai K, et al. Diagnostic values of soluble mesothelinrelated peptides for malignant pleural mesothelioma: updated metaanalysis. BMJ Open 2014;4:e004145.

7 Bibby AC, Tsim S, Kanellakis N, et al. Malignant pleural mesothelioma: an update on investigation, diagnosis and treatment. Eur Respir Rev 2016;25:472-86.

8 Johnen G, Burek K, Raiko I, et al. Prediagnostic detection of mesothelioma by circulating calretinin and mesothelin - a casecontrol comparison nested into a prospective cohort of asbestosexposed workers. Sci Rep 2018;8:14321.

9 Pesch B, Brüning T, Johnen G, et al. Biomarker research with prospective study designs for the early detection of cancer. Biochim Biophys Acta 2014;1844:874-83.

10 Beyer HL, Geschwindt RD, Glover CL, et al. MESOMARK: a potential test for malignant pleural mesothelioma. Clin Chem 2007;53:666-72.

11 Hollevoet K, Bernard D, De Geeter F, et al. Glomerular filtration rate is a confounder for the measurement of soluble mesothelin in serum. Clin Chem 2009;55:1431-3.

12 Weber DG, Johnen G, Taeger D, et al. Assessment of confounding factors affecting the tumor markers SMRP, CA125, and CYFRA21-1 in serum. Biomark Insights 2010;5:1-8.

13 Casjens S, Weber DG, Johnen G, et al. Assessment of potential predictors of calretinin and mesothelin to improve the diagnostic performance to detect malignant mesothelioma: results from a population-based cohort study. BMJ Open 2017;7:e017104.

14 Casjens S, Pesch B, Robens S, et al. Associations between former exposure to manganese and olfaction in an elderly population: results from the heinz Nixdorf recall study. Neurotoxicology 2017;58:58-65.

15 Schmermund A, Möhlenkamp S, Stang A, et al. Assessment of clinically silent atherosclerotic disease and established and novel risk factors for predicting myocardial infarction and cardiac death in healthy middle-aged subjects: rationale and design of the heinz Nixdorf recall study. risk factors, evaluation of coronary calcium and lifestyle. Am Heart J 2002;144:212-8.

16 Eisele L, Dürig J, Broecker-Preuss M, et al. Prevalence and incidence of anemia in the German heinz Nixdorf recall study. Ann Hematol 2013;92:731-7.

17 Raiko I, Sander I, Weber DG, et al. Development of an enzyme-linked immunosorbent assay for the detection of human calretinin in plasma and serum of mesothelioma patients. BMC Cancer 2010;10:242.

18 Scholler N, Fu N, Yang Y, et al. Soluble member(s) of the mesothelin/ megakaryocyte potentiating factor family are detectable in sera from patients with ovarian carcinoma. Proc Natl Acad Sci U S A 1999;96:11531-6.

19 Tsim S, Kelly C, Alexander L, et al. Diagnostic and prognostic biomarkers in the rational assessment of mesothelioma (diaphragm) study: protocol of a prospective, multicentre, observational study. BMJ Open 2016;6:e013324.

20 Lehnert M, Kraywinkel K, Heinze E, et al. Incidence of malignant mesothelioma in Germany 2009-2013. Cancer Causes Control 2017;28:97-105.

21 Filler G, Bökenkamp A, Hofmann W, et al. Cystatin C as a marker of GFR--history, indications, and future research. Clin Biochem 2005;38:1-8.

22 Ma Y, Li Q, Wang J, et al. Cystatin C, a novel urinary biomarker for sensitive detection of acute kidney injury during haemorrhagic fever with renal syndrome. Biomarkers 2010;15:410-7.

23 Okamoto R, Ali Y, Hashizume R, et al. Bnp as a major player in the Heart-Kidney connection. Int J Mol Sci 2019;20:ijms20143581. doi:10.3390/ijms20143581

24 Tsolaki V, Zarogiannis S, Zygoulis P, et al. Malignant mesothelioma cells secrete natriuretic peptides: data and diagnostic clinical implications. Respirology 2020;25:1060-5.

25 Diederichs $\mathrm{C}$, Neuhauser $\mathrm{H}$. The incidence of hypertension and its risk factors in the German adult population: results from the German National health interview and examination survey 1998 and the German health interview and examination survey for adults 20082011. J Hypertens 2017;35:250-8.

26 Jiménez-Ramírez C, Casjens S, Juárez-Pérez CA, et al. Mesothelin, calretinin, and megakaryocyte potentiating factor as biomarkers of malignant pleural mesothelioma. Lung 2019;197:641-9. 
27 Pass HI, Wali A, Tang N, et al. Soluble mesothelin-related peptide level elevation in mesothelioma serum and pleural effusions. Ann Thorac Surg 2008;85:265-72.

28 Weber DG, Taeger D, Pesch B, et al. Soluble mesothelin-related peptides (SMRP) - high stability of a potential tumor marker for mesothelioma. Cancer Biomark 2007;3:287-92.

29 Felten MK, Khatab K, Knoll L, et al. Changes of mesothelin and osteopontin levels over time in formerly asbestos-exposed power industry workers. Int Arch Occup Environ Health 2014;87:195-204.
30 Robinson BWS, Creaney J, Lake R, et al. Mesothelin-family proteins and diagnosis of mesothelioma. Lancet 2003;362:1612-6.

31 Davies HE, Sadler RS, Bielsa S, et al. Clinical impact and reliability of pleural fluid mesothelin in undiagnosed pleural effusions. $A m \mathrm{~J}$ Respir Crit Care Med 2009;180:437-44.

32 Foddis R, Bonotti A, Landi S, et al. Biomarkers in the prevention and follow-up of workers exposed to asbestos. J Thorac Dis 2018;10:S360-8. 